\title{
Condição de saúde bucal de crianças internadas no Hospital Municipal Infantil de Imperatriz - Maranhão
}

\author{
Oral health status of children admitted to the Children's Municipal Hospital of Imperatriz-Maranhão
}

Márcia Cristina Pereira de Souza Lima

Professora da Faculdade de Imperatriz (FACIMP) Mestra em Odontopediatria pela Universidade Cruzeiro do Sul (UNICSUL)

Ingrid Natachilla Rodrigues Lobo

Faculdade de Imperatriz (FACIMP)

Especializanda em Implantodontia pela ABO-MA

Katiane Vieira Menezes Leite

Professora da FACIMP

Mestra em Prótese pela São Leopoldo Mandic - SP

Gabrielle Ribeiro Lima Muniz

Professora da FACIMP

Mestra em Odontologia pela Universidade Federal do Maranhão (UFMA)

Henrique Caballero Steinhauser

Professor da FACIMP

Mestre em Prótese pela São Leopoldo Mandic - SP

Paulo Roberto Martins Maia

Professor da FACIMP

Especialista em Ortodontia pela UFMA

\section{Resumo}

O objetivo deste artigo foi avaliar a condição de saúde bucal de crianças internadas no Hospital Municipal Infantil de Imperatriz - MA. A amostra foi constituída por 80 crianças de ambos os sexos, com idade entre 02 e 12 anos, hospitalizadas por no mínimo 03 dias. Quanto à distribuição da média do ceo-d e CPO-D por idade, as crianças hospitalizadas de 05 a 07 anos apresentaram índice de cárie mais elevado. $0 \mathrm{IHOS}$, na faixa etária entre 02 e 05 anos, revelou uma higiene bucal insatisfatória. Constatouse que a saúde bucal das crianças hospitalizadas está comprometida, com um alto índice de cárie na dentição decídua. A higiene bucal não ocorre nem de forma sistemática, nem orientada durante o período de hospitalização, havendo uma baixa adesão aos procedimentos de higiene bucal.

Palavras-chave: saúde bucal; cárie dental; prevalência.

\section{Abstract}

The aim of this paper was to evaluate the oral health status of children hospitalized at Children's Municipal Hospital Imperatriz - MA. The sample consisted of 80 children of both sexes, aged between 02 to 12 years hospitalized for at least 03 days. As for the distribution of the average ceo- $d$ and CPOD age, hospitalized children 05 to 07 years had higher caries prevalence. The IHOS, aged between 02 to 05 , revealed a poor oral hygiene. It was found that the oral health of hospitalized children is committed, with a high index of caries in the primary dentition. The oral hygiene does not occur neither systematically nor targeted during the hospitalization period, with a low adherence to oral hygiene procedures.

Keywords: oral health; dental caries; prevalence.

Agradecimentos

Hospital Municipal Infantil de Imperatriz (HMII)

\section{Introdução}

A boca é parte integrante do corpo e interfere decisivamente no aparecimento e disseminação de patologias. Na cavidade bucal existem mais de 300 espécies de bactérias responsáveis por patologias bucais como a cárie e doença periodontal e/ou sistêmicas quando atingem sítios como coração, pulmões, articulações e sistema vascular periférico (1-5).

De acordo com o Ministério da Saúde (6), a cárie dentária é a patologia bucal mais comum no mundo, afetando cinco bilhões de pessoas, ou cerca de $80 \%$ da população mundial. No Brasil, $88 \%$ da população são acometidas por cárie, colocando este entre os países com mais problemas bucais.

Apesar da doença cárie ser a mais prevalente, outras alterações também são observadas, estudo feito na cidade de São Luis - MA, avaliou clinicamente as alterações da mucosa bucal de 165 crianças de 03 a 12 anos hospitalizadas, das quais 112 (67,88\%) apresentaram algum tipo de alteração de mucosa, demonstrou que as alterações mais frequentes foram língua saburrosa $(61,82 \%)$, candidíase pseudomembranosa $(5,45 \%)$, infecção herpética recorrente $(3,64 \%)$ e estomatite aftosa recorrente (3,03\%) (7).

Além da vulnerabilidade sistêmica como fator relacionado ao desenvolvimento de patologias bucais, fatores determinantes da doença cárie e da doença periodontal estão presentes na rotina hospitalar e se tornam mais significativos com aumento do tempo de internação. Estes fatores referem-se à cariogenicidade da dieta, utilização de medicação sob a forma de xaropes, falta de disposição para a realização da higiene bucal e falta de motivação dos pais e/ou cuidadores sobre os benefícios que os cuidados em saúde bucal trarão para a criança $(8,9)$.

De acordo com o Sistema de Informações Hospitalares do Sistema Único de Saúde (SIH/SUS) (10), no ano de 2014, na especialidade de pediatria, foram realizadas 677.803,04 internações, na cidade de Imperatriz, Maranhão.

Durante a hospitalização, a criança está sujeita a uma série de fatores muito diferentes aos da sua rotina habitual que podem contribuir negativamente para a sua saúde bucal. A criança, diante do internamento hospitalar, responde de maneira diversificada, com várias alterações bio psico comportamentais, podendo ser uma das maiores fontes de ansiedade e estresse para as mesmas, como também para pais e familiares, trazendo comportamentos como apatia e tristeza (11).

Além do estresse da hospitalização e da própria doença, a criança tem que lidar com o ambiente hostil: alto nível de ruído, perda do sono, luzes intensas, procedimentos às vezes imprevisíveis e mudança drástica da rotina normal. Em adição a isso, a mudança nos horários das refeições e nos hábitos alimentares; a introdução de medicamentos na rotina diária; a indisposição ocasionada pela doença e a estadia em um ambiente diferente do habitual levam à subvalorização dos cuidados bucais (12).

Consequentemente, esta falta de higienização pode promover o acúmulo de biofilme dentário, estrutura diretamente relacionada a vários problemas bucais, tais como a cárie e doenças periodontais. Sendo assim, faz-se necessário o acompanhamento dos cuidados com a saúde bucal de crianças hospitalizadas visando sua manutenção (13-16). 
A partir de 03 dias, crianças hospitalizadas apresentam um índice de placa médio de $67,7 \%$, a partir de 05 dias esse valor chega a $100 \%$ (17).

A relação existente entre antibacterianos e cárie dentária envolve uma série de fatores, dentre os mais amplamente descritos na literatura pode-se destacar: a presença de sacarose nas formulações destes fármacos, o baixo $\mathrm{pH}$ de algumas formulações e o uso frequente por crianças portadoras de doenças crônicas tais como asma, otite média recorrente e infecção do trato respiratório superior (18-20).

Desse modo, crianças com doenças crônicas que fazem uso contínuo de medicamentos contendo sacarose, na forma líquida ou comprimido mastigável, com administrações repetidas e algumas em períodos de sono, podem apresentar risco ao desenvolvimento de cárie dentária se houver ausência de higiene bucal após a administração dos mesmos (21-23).

Geralmente os pais ou responsáveis por crianças hospitalizadas não recebem orientações da equipe de saúde sobre a correta higiene bucal após a ingestão de medicamentos e, quando esta ocorre, não é feita por um cirurgião-dentista (24-29).

Em um estudo realizado sobre o perfil da assistência odontológica na infância e adolescência na cidade de São Luís, Maranhão, demonstrou que a atenção odontológica tem sido mais voltada para a dentadura permanente do que para a decídua (30).

Devido à relevância deste tema, o presente estudo se propôs a avaliar e discutir as condições de saúde bucal de crianças hospitalizadas, bem como estabelecer sua relação com as características da internação visando à obtenção de dados para orientar na formulação de estratégias de ação do cirurgião-dentista neste espaço diferenciado.

\section{Material e Métodos}

Este estudo foi realizado no Hospital Municipal Infantil de Imperatriz - MA, único hospital público destinado ao atendimento de pacientes de 0 a 12 anos de idade, de referência terciária do SUS, caracteriza-se como uma unidade pública de média complexidade, de referência municipal e regional para o atendimento do público infantil, oferta uma gama de serviços ambulatoriais, procedimentos cirúrgicos e internação hospitalar.

A amostra desta pesquisa foi composta por 80 crianças de 02 a 12 anos de idade, de ambos os sexos, representadas por seus responsáveis, admitidas no setor de internação do Hospital Municipal Infantil de Imperatriz - MA.

Não foi realizado cálculo amostral, tendo em vista que a pesquisa foi realizada em ambiente hospitalar, não tendo, portanto, previsibilidade da permanência de pacientes, que dependiam da melhora de seu quadro clínico, para receberem alta médica. Sendo assim, a amostra foi composta pelo número de crianças que estavam presentes no momento da pesquisa e que se enquadraram nos critérios de inclusão que foram: ser pacientes internados em um período de tempo igual ou superior a 03 dias e idade entre 02 a 12 anos; concordar em participar da amostra; seu responsável ter sido informado sobre a pesquisa e ter assinado o termo de consentimento livre e esclarecido. Justifica-se a escolha deste período de tempo, pois se espera que após o mesmo o paciente já tenha seu quadro clínico estabilizado como também adaptado à rotina hospitalar. A escolha da idade, a partir de dois anos, foi feita com base na cronologia da irrupção dentária, a qual afirma que por volta 24-30 meses de idade a criança já se encontra com todos os elementos dentários decíduos irrompidos (14), favorecendo assim a realização dos exames clínicos.

Para a coleta de dados, foi realizada primeiramente uma triagem dos pacientes através da análise de seus prontuários. Foram selecionados os pacientes na referida faixa etária e que estavam hospitalizados a 03,06, 09 e mais de 09 dias, com a finalidade de verificar a influência do fator tempo aliado às características da internação hospitalar nas condições de saúde bucal.

Em seguida, os dados sociodemográficos e avaliação da saúde bucal foram registrados em ficha própria. Foram investigados, hábitos comportamentais durante o período de internação como, adoção de práticas de higiene bucal, e quais instrumentos utilizados para a realização da higiene bucal.

Foi questionado aos responsáveis pelas crianças quanto a sua opinião acerca da relação da ingestão de medicamentos açucarados e o desenvolvimento de cárie dentária, se receberam orientações de higiene bucal concedida por profissionais da unidade hospitalar, se já haviam recebido orientações de higiene bucal concedida por médico ou cirurgião dentista fora da unidade hospitalar e se as crianças já haviam tido experiência odontológica.

Os dados referentes à internação como: motivo da hospitalização, episódio de internação anterior, medicação administrada e tipo de dieta foram obtidos a partir dos respectivos prontuários médicos no posto de enfermagem.

O exame da cavidade bucal foi realizado para a avaliação da saúde bucal no final da entrevista, utilizando-se espátula de madeira, no próprio ambiente hospitalar.

Os exames bucais foram realizados por uma única examinadora, previamente calibrada, com verificação da concordância através da estatística Kappa 0,98. Para definição da condição bucal das crianças, utilizou-se os índices ceo-d e CPO-D, para dentes decíduos e dentes permanentes, respectivamente. Para dentes permanentes " $\mathrm{C}$ " é o número de dentes cariados, "P" para dentes perdidos e "O" para os obturados (restaurados). O índice ceo-d para dentes decíduos, onde "c" indica o número de dentes cariados, "e" os dentes com extração indicada e "o" os dentes obturados (restaurados).

Para a realização do exame da cavidade bucal, utilizaram-se os equipamentos de proteção individual, como máscara, gorro, óculos e luvas. O exame clínico foi realizado de forma sistemática e ordenada, utilizando os números de dois dígitos para dentes específicos de acordo com o sistema 
utilizado pela Federação Dentária Internacional - FDI. As superfícies dentárias foram secas com auxílio de gaze estéril (BRASIL, 2010).

Posteriormente foi avaliado, o Índice de Higiene Oral Simplificado (IHO-S), onde apenas os elementos dentários totalmente irrompidos foram considerados e na ausência de um elemento dentário este foi substituído por outro elemento do mesmo grupo. A cada superfície evidenciada foi atribuído um código que varia de 0 a 3 para o biofilme: 0 para a ausência de biofilme, 1 para $1 / 3$ da face evidenciada, 2 para $2 / 3$ da face evidenciada e 3 para a face que for totalmente evidenciada. A evidenciação do biofilme dental foi realizada com solução evidenciadora a base de fucsina utilizando-se microaplicadores odontológicos descartáveis para espalhar a substância nas superfícies dentárias, tendo auxílio de luz artificial e espelho clínico. Após o exame clínico intrabucal, foi realizada instruções de higiene bucal para a criança e seu responsável e doação de escova dentária.

$\mathrm{Na}$ análise dos dados foram obtidas distribuições absolutas e percentuais bivariadas (técnicas de estatística descritiva). Os dados foram digitados e tabulados no programa Office Excel for Windows e as estatísticas foram feitas no programa SPSS (Statistical Package for the Social Sciences) na versão 20 .

Esta pesquisa foi submetida e aprovada pelo Comitê de Ética em Pesquisa da Faculdade de Imperatriz (FACIMP) MA, sob o protocolo n.016/2013.

\section{Resultados}

Dentre as variáveis relacionadas à caracterização do grupo das crianças, observamos que $63,75 \%(n=51)$ eram do sexo masculino e $36,25 \%(n=29)$ do sexo feminino. A média de idade das crianças foi de 5,8 anos, situadas no grupo da primeira infância período que compreende do primeiro ao sexto ano de vida. Ao verificar os motivos da internação, as doenças infecciosas e parasitárias foram as que mais acometeram as crianças hospitalizadas (37,5\%). O período de internação hospitalar de maior prevalência durante a pesquisa foi de 03 dias (57,50\%). Observou-se também que $57,50 \%$ das crianças já haviam passado por episódios de internações anteriores, enquanto que $42,50 \%$ nunca haviam sido internadas.

Observou-se que os horários com maiores frequências de consumo de medicação cariogênica foram: às $6 \mathrm{~h}$ (35\%), seguido dos horários das $12 \mathrm{~h}(32,5 \%)$ e $24 \mathrm{~h}(32,5 \%)$. Destacou-se que entre as crianças examinadas $46,25 \%$ recebiam medicação por via oral. Entre as classes terapêuticas mais citadas elencamos: os anti-inflamatórios, analgésicos e antipiréticos $(16,25 \%)$, antisséptico intestinal $(8,75 \%)$, seguidos dos anti-infecciosos e probióticos (7,5\%), broncodilatadores e expectorantes $(7,5 \%)$, a maioria sob a forma de gotas.

Pesquisou-se também o tipo de dieta oferecida às crianças hospitalizadas, destacou-se a dieta livre $(72,50 \%)$ como a mais empregada. O cardápio consistia em biscoito, pão, leite, mingau com açúcar, suco com açúcar, inter- calado a cada 03 horas.

Analisando os hábitos de higiene bucal das 80 crianças avaliadas durante o período de hospitalização, constatamos que a maioria delas, $(75 \%)$ realizavam a higiene bucal durante a hospitalização. Dentre essas que praticavam a higiene bucal, quem a realizava na maioria das vezes era a própria criança $32,50 \%$; nas demais, essa higienização era realizada pelo adulto $29 \%$, e pelas crianças auxiliadas pelos adultos $13,50 \%$. A maioria utilizava escova e creme dental $(73,75 \%)$, apenas uma criança $(1,25 \%)$ havia utilizado o fio dental durante esse período.

Quanto à frequência da higiene bucal durante a hospitalização, 43,75\% ( $\mathrm{n}=35)$ relataram realizar a higiene bucal uma vez ao dia, $22,50 \%(n=18)$ duas vezes ao dia e somente $8,75 \%(\mathrm{n}=7)$ declararam realizar a higiene bucal três ou mais vezes ao dia (Figura 1).

Quanto à qualidade da escovação dental, observamos que com 03 dias de internação, 65,2\% das crianças apresentavam uma higiene bucal insatisfatória, verificada pelo IHOS, permanecendo quase o mesmo percentual para aquelas que estavam hospitalizadas a mais de 09 dias $(62,5 \%)$ (Figura 2).

Sobre a higiene bucal noturna, $76,25 \%$ relataram que nunca a realizava, seguido de $17,50 \%$ que afirmaram realizar todos os dias, $5 \%$ frequentemente e 1,25\% raramente (Figura 3).

Indagou-se aos pais ou responsáveis pelas crianças hospitalizadas, em relação às orientações de higiene bucal obtidas durante a internação, 98,75\% ( $\mathrm{n}=79)$ dos responsáveis informaram que não haviam recebido orientações durante o período em que as crianças permaneciam hospitalizadas; o único responsável, que recebeu algum tipo de orientação $(1,25 \%)$, foi concedida por uma enfermeira (Figura 4).

Quanto à distribuição da média do ceo-d e CPO-D por idade (Figura 5), revelou que as crianças hospitalizadas na faixa etária entre 05, 06 e 07 anos são as que apresentaram índice de cárie alto com ceo- $\mathrm{d}=5,14,6,0$ e 6,40, respectivamente. As demais idades revelaram um índice de cárie muito baixo $\mathrm{CPO}-\mathrm{D}=0,64$ a 0,28 .

Quanto à distribuição da média do IHOS dos pacientes hospitalizados (gráfico 6), observamos que na faixa etária de 2 a 5 anos a média do índice de placa foi maior (entre 2,2 à 2,1), revelando uma higiene bucal insatisfatória, neste grupo de crianças. Entre as idades de 6 a 12 anos observou-se um declínio da média do índice.

A presente pesquisa objetivou avaliar a condição de saúde bucal de crianças hospitalizadas no Hospital Municipal da cidade de Imperatriz, Maranhão. Pode-se verificar que $25 \%$ das crianças hospitalizadas não realizaram procedimentos de higiene bucal durante o período de internação, outras pesquisas também tem revelado uma baixa adesão a essa prática. Isto é preocupante, visto que crianças hospitalizadas apresentam debilidade sistêmica, se estas práticas não estiverem presentes na rotina hospitalar, pode comprometer e agravar a condição bucal e sistêmica das crianças $(1,9,29)$. 
É necessário que os cuidados com a higiene bucal sejam proporcionados em uma base diária e regular, por isso procurou-se verificar se as crianças hospitalizadas mantinham uma frequência de escovação satisfatória. Demonstrou-se que a maioria das crianças hospitalizadas não mantinham uma higiene com uma frequência regular, grande parte $(43,74 \%)$ realizava a higiene bucal apenas uma vez por dia. Resultados semelhantes foram apresentados por Ximenes et al. (2008), que verificaram que os pacientes em hospitais ou em instituições que necessitem de tratamento de longo prazo geralmente não recebem o cuidado intensivo de que precisam, isto é algo preocupante visto que se trata de crianças sob a responsabilidade de uma equipe de saúde, que deveria fornecer todo o suporte para a manutenção da saúde da criança.

Das crianças hospitalizadas que realizavam a higiene bucal, $73,75 \%$ utilizavam a escova e o creme dental, porém o uso do fio dental só foi registrado por uma criança $(1,25 \%)$, pesquisas tem demonstrado que o uso do fio dental não é um hábito na população infantil, em uma pesquisa realizada com crianças hospitalizadas, foi observado que 90,6\% das crianças que realizavam higiene bucal utilizavam escova e o creme dental, porém não foi registrado o uso do fio dental (9). Não há na atualidade, recurso mais seguro para o controle da placa bacteriana que os representados pela escova dental para as faces livres dos dentes e o fio dental para as faces proximais, corroborado pelos achados de GUEDES-PINTO (13).

O presente estudo revelou que orientações sobre prática de higiene bucal são raramente abordadas pela equipe de saúde que presta assistência à criança hospitalizada, uma vez que apenas um responsável $(1,25 \%)$ relatou ter recebido algum tipo de orientação sobre o assunto durante o período de internação. A maioria dos responsáveis pela criança não recebem orientações sobre higiene bucal durante o período de internação, e quando os responsáveis recebem algum tipo de orientação, essas são concedidas por médicos, enfermeiros e técnicos de enfermagem (29). Em um estudo realizado com usuários na Unidade de Internação Pediátrica do Hospital Universitário da Universidade Federal de Santa Catarina, a presença do cirurgião-dentista no ambiente hospitalar revelou-se como uma novidade, mas ficou evidente a valorização do profissional da odontologia, uma vez que os participantes da pesquisa reconheceram que o cirurgião-dentista é o profissional especializado para cuidar da saúde bucal (27).

A presente pesquisa constatou também que apenas $27,50 \%$ das crianças hospitalizadas tinham experiência odontológica, revelando um reduzido acesso do público infantil à assistência odontológica. Estudos tem revelado que a atenção odontológica é mais voltada para a dentição permanente, o que denota maior carência de cuidados odontológicos em crianças de menor idade, ou na primeira infância $(29,30)$.

Com relação ao período de hospitalização, foi observado que $57,50 \%$ das crianças encontravam-se hospitalizadas por um período de três dias. No entanto, algumas crianças estavam internadas por um período maior, sem a realização de qualquer cuidado com a saúde bucal com o consumo de uma dieta cariogênica e medicamentos contendo açúcar em horários considerados de risco, por um tempo que já permitiria a instalação de um processo carioso ou gengivite, fatos semelhantes foram encontrados por XIMENES, ARAGÃO, COLARES (9), SILVEIRA et al. (11), LIMA (12). Na maioria das internações hospitalares de crianças, o tempo de internação varia de 03 a mais dias, assim, as crianças hospitalizadas estão submetidas a desafios cariogênicos constantes em razão da ingestão de medicamentos açucarados, por longos períodos, os quais propiciam a desmineralização no esmalte dentário e posteriormente a formação de cárie dentária. Esses medicamentos desencadeiam dois efeitos importantes na etiologia da cárie dentária: o primeiro é que na forma líquida, contém sacarose, o que provoca um $\mathrm{pH}$ muito baixo, ou seja, a formação dos ácidos na interface biofilme-esmalte. $\mathrm{O}$ segundo efeito é o potente efeito redutor do fluxo salivar, importante na autolimpeza bucal, no equilíbrio biológico das bactérias, na neutralização dos ácidos do biofilme dentário e no processo desremineralização $(17,19,21)$.

Em se tratando de biofilme dental, o presente estudo observou que na faixa etária entre 02 e 05 anos, a média do índice de placa foi maior (IHOS entre 2,2 a 2,1), revelando uma higiene bucal insatisfatória neste grupo de crianças, apontando que quanto mais nova a criança, pior é a qualidade de higiene bucal. Fatos também descritos por PRADO et al. (31) e SOARES et al. (32). Observamos que a qualidade de higiene bucal com os dias de hospitalização era insatisfatória com 03 dias de hospitalização (variando de $65,2 \%$ a 100\%). Fatos semelhantes foram relatados por AMARAL et al. (17).

Alguns fatores são relacionados à higiene bucal insatisfatória em crianças hospitalizadas: a faixa etária (os menores não tem coordenação motora suficiente para uma escovação adequada), a própria situação em que se encontram (falta de disposição física, devido à debilidade do organismo), a falta e motivação dos pais e das crianças sobre os benefícios da higiene bucal ou mesmo, os conhecimentos e práticas da equipe de saúde hospitalar sobre saúde $(11,19)$.

Em relação ao índice de cárie das crianças hospitalizadas, a presente pesquisa revelou valores mais altos na dentição decídua, nas crianças entre a faixa etária de 05 e 07 anos. Estudos tem explicado os fatores que podem estar relacionados aos valores de ceo-d mais altos que o CPO-D, como a negligência que ocorre na adoção das práticas de higiene bucal em crianças de menor idade, além disso, para alguns pais a dentição decídua não é considerada tão importante quanto a permanente (15).

Quando foi questionado aos pais ou responsáveis pela criança, se já haviam recebido orientações por médico ou dentista para a realização da higiene bucal após a ingestão de medicamentos líquidos, apenas $2,50 \%$ responderam sim, sendo estas orientações repassadas por um médico e por um dentista, $97,50 \%$ referiu não ter recebido nenhum tipo de 
orientação. A equipe de saúde do hospital não transmite recomendações de higiene bucal pós-consumo de medicamentos açucarados (19).

É importante salientar que as instruções fornecidas por cirurgiões-dentistas quanto à importância da higienização bucal de seus filhos após a ingestão de medicamentos, repercutem positivamente, pois os pais ou responsáveis tendem a aplicar essas informações (25).

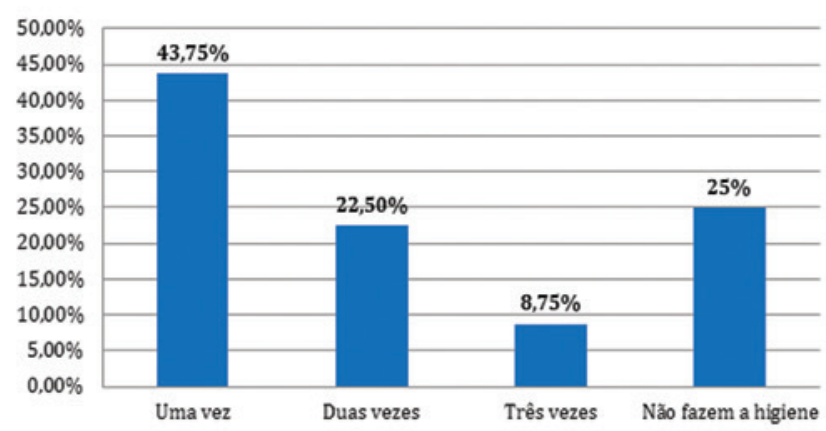

Figura 1. Percentual da frequência de higiene bucal das crianças hospitalizadas. HMII, Imperatriz- MA, 2013

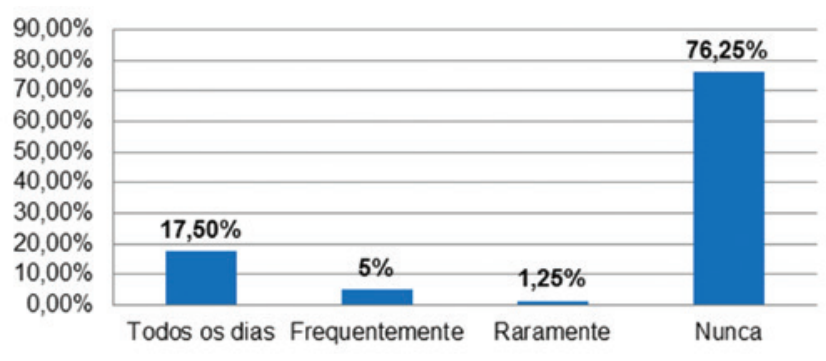

Figura 3. Percentual quanto à realização de higiene bucal noturna das crianças hospitalizadas. HMII, Imperatriz- MA, 2013

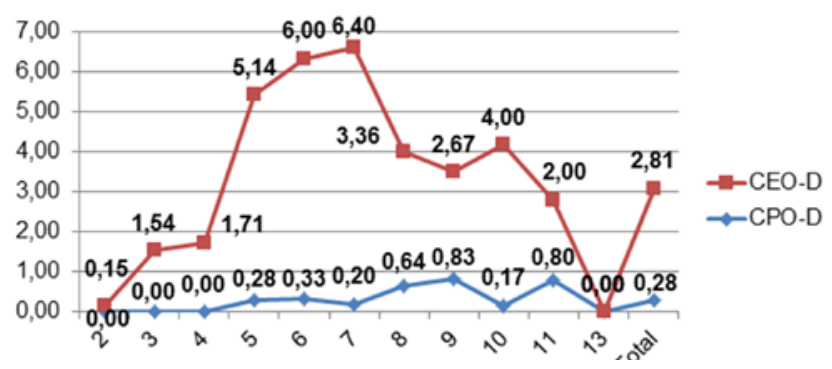

Figura 5. Distribuição da média do ceo-d e CPO-D por idade de 2 a 12 anos das crianças hospitalizadas. HMII, Imperatriz- MA, 2013

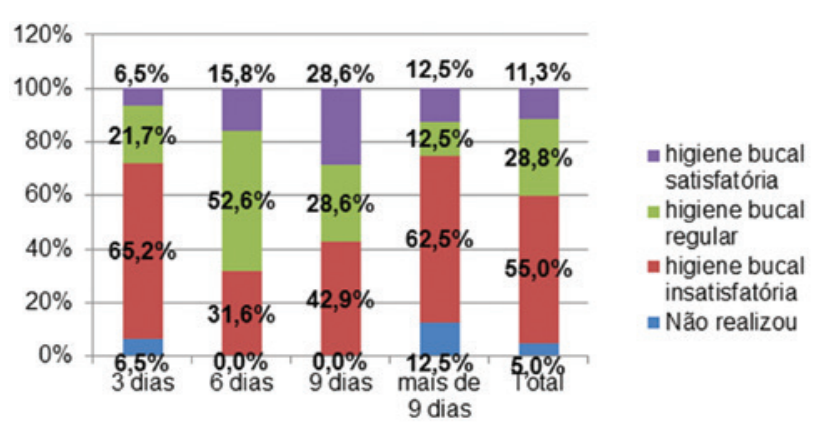

Figura 2. Percentual da associação entre a classiticação da higiene bucal com o tempo de internação das crianças hospitalizadas. HMII, Imperatriz- MA, 2013

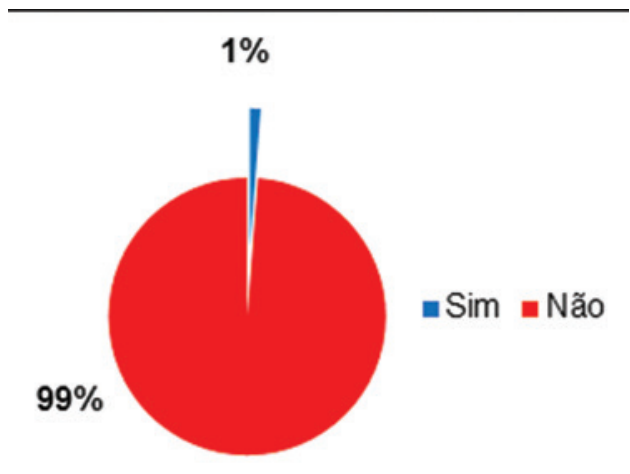

Figura 4. Percentual de responsáveis pelas crianças hospitalizadas que receberam orientações sobre higiene bucal por profissionais da unidade hospitalar. HMII, Imperatriz- MA, 2013

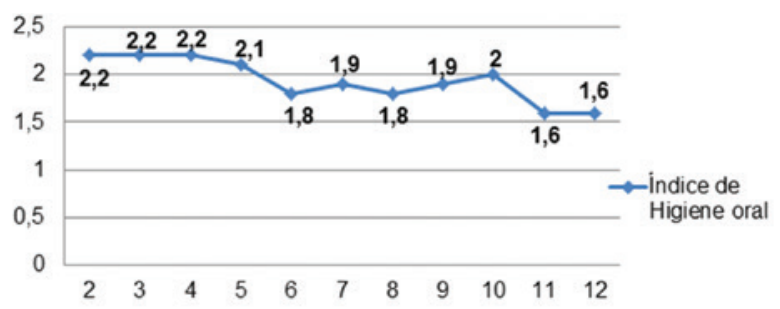

Figura 6. Distribuição da média do índice de higiene oral simplificado (IHO-S) por idade, de 2 a 12 anos das crianças hospitalizadas. HMII, Imperatriz- MA, 2013

\section{Conclusão}

Frente aos resultados encontrados, conclui-se que a condição de saúde bucal das crianças hospitalizadas está comprometida por um alto índice de biofilme dental e um alto índice de cárie na dentição decídua. A higiene bucal não ocorre de forma sistemática e orientada durante a hospitalização, havendo uma baixa adesão aos procedimentos de higiene, um reduzido acesso à assistência odontológica, enquanto a higiene bucal é desvalorizada no contexto da criança hospitalizada.

Sendo assim, sugere-se a necessidade de se problematizar a abordagem da saúde bucal em ambiente hospitalar. Deve ser estimulada a participação de um odontopediatra na equipe multidisciplinar de saúde nos hospitais, no intuito de enfatizar a adoção de ações de saúde bucal de maneira a contribuir com a saúde geral das crianças hospitalizadas, sendo essencial durante esse processo, envolver todos os sujeitos relacionados aos cuidados: criança, responsáveis e profissionais da saúde. 


\section{Referências ::}

1. Almeida FR, et al. Associação entre doença periodontal e patologias sistêmicas. Rev. Port. Clín. Geral. 2006;22:370-90.

2. Ribeiro BB, et al. Importância do reconhecimento das manifestações bucais de doenças e de condições sistêmicas pelos profissionais de saúde com atribuição de diagnóstico. Odonto. 2012;2:1-10.

3. Roriz VM, Barbosa RA. Possibilidade de inter-relação entre as doenças periodontais e as cardiovasculares. Rev. Odontol. Bras. Central. 2011;20(55).

4. Oliveira FJ. Inflamação sistêmica causada pela periodontite crônica em pacientes vítimas de ataque cardíaco isquêmico agudo. Rev. Bras. Cir. Cardiovasc. 2010;25(1):51-8.

5. Vieira TR, Peret ACA, Filho LAP. Alterações periodontais associadas às doenças sistêmicas em crianças e adolescentes. Rev. Paulista de Pediatr. 2010;28(2):237.

6. Brasil. Ministério da Saúde. Projeto SB Brasil 2010: Pesquisa Nacional de Saúde Bucal 2010. Resultados principais. Brasília: Ministério da Saúde; 2011.

7. Cruz MCFN, Valois EM, Libério SA, et al. Avaliação clínica das alterações de mucosa bucal em crianças hospitalizadas de 3 a 12 anos. RGO. 2008;56(2):157-61.

8. Silva MJCN, et al. Por que devemos nos preocupar com a saúde bucal de crianças hospitalizadas? Interagir: pensando a extensão. 2009;14:17-20.

9. Ximenes RCC, Aragão DSF, Colares V. Avaliação dos cuidados com a saúde oral de crianças hospitalizadas. Rev. Fac. Odontol. 2008;49(1).

10. Brasil. Ministério da Saúde. Sistema de Informações Hospitalares do SUS (SIH/SUS) [Internet]. [acesso em 2015 fev. 20]. Disponível em: http:// tabnet.datasus.gov.br/cgi/deftohtm.exe?sih/cnv/sxsc.def

11. Silveira ER, et al. Condição de saúde bucal de crianças internadas no hospital escola da Universidade Federal de Pelotas- RS. XIII ENPOS. 2011.

12. Lima LS. Fatores associados à condição de saúde bucal de pacientes internados em hospitais públicos do município de Natal- RN. Rev. Fac. Odontol. Natal, RN. 2007.

13. Guedes-Pinto AC. Odontopediatria. 7. ed. São Paulo: Liv. Santos; 2006 .

14. Teixeira KIR, Bueno CA, Cortez ME. Processos físico-químicos no biofilme dentário relacionados à produção da cárie. Química Nova Escola. 2010;32(3).

15. Tomita NE, Bijella VT, Lopes ES, et al. Prevalência de cárie dentária em crianças da faixa etária de 0 a 6 anos matriculadas em creches: importância dos fatores sócio-econômicos. Rev. Saúde Pública. 1996;30(5):413-20.

16. Pereira AC. Tratado de Saúde Coletiva em Odontologia. São Paulo. Napoleão. 2009;288.

17. Amaral KC, Tenório MDH, Dantas AB. Condição de saúde bucal de crianças internas em hospitais da cidade de Maceió-AL. Odontologia. Clín. - Científ. 2006;5:267-73.
18. Sahgai J, Sood PB, Raju OS. A comparison of oral hygiene status and dental caris in children on long term liquid oral medications to those not administered with such medications. J Indian Soc Pedod Prev Dent. 2002.

19. Leite F, Leite C, Pinto ME. Medicamentos pediátricos e cáries dentárias- Percepções e atitudes de um grupo de tutores pediátricos em Vila Nova de Gaia. Revista Portuguesa de Estomatologia, Medicina Dentária e Cirurgia Maxilofacial. 2011;52(4):193-9.

20. Neiva A, et al. Análise in vitro da concentração de sacarose e ph deantibacterianos de uso pediátrico. Pesquisa Brasileira em Odontopediatria e Clínica Integrada. 2001;1(1).

21. Passos IA, Freitas CHSM, Sampaio FC. Potencial cariogênico de medicamentos pediátricos - papel na etiologia da cárie dentária. Rev. Inst. Ciênc. Saúde. 2008;26(1):125-9.

22. Costa CC, et al. Análise do pH endógeno, da acidez e da concentração de sacarose de medicamentos pediátricos. Rev. Odonto. Ciênc. 2004.

23. Morais TMN, Silva A, Avir ALR, et al. A importância da atuação odontológica em pacientes internados em unidade de terapia intensiva. Revista Brasileira de Terapia Intensiva. 2006;18(4).

24. SILVA, NVA. Doenças exantêmicas da infância e suas manifestações orais. Faculdade de Medicina dentária- Universidade do Porto. Porto. 2010.

25. Neves BG, Pierro VSS, Maia IC. Percepções e atitudes de responsáveis por crianças frente ao uso de medicamentos infantis e sua relação com cárie e erosão dentária. Rev. Ciência e Saúde Coletiva. 2007;12(5):1295-300.

26. Losso EM, et al. Cárie precoce e severa na infância: uma abordagem integral. J. pediatr. 2009;85(4):295-300.

27. Mattevi GS, Figueiredo DR, Patricio ZM, et al. A participação do cirurgião-dentista em equipe de saúde multidisciplinar na atenção à saúde da criança no contexto hospitalar. Ciênc. saúde coletiva [online]. 2011;16(10):4229-36.

28. Dias J. Saúde bucal de crianças hospitalizadas- o cuidado das enfermeiras pediatras. Universidade Federal do Rio Grande do Sul. Porto Alegre. 2010.

29. Rodrigues VP, et al. Avaliação dos hábitos de higiene bucal de crianças durante o período de internação hospitalar. Odontol. Clín. Cient. 2011;10(1):49-55.

30. Silva MCB, Silva RA, Ribeiro CCC, et al. Perfil da assistência odontológica pública para a infância e adolescência em São Luís (MA). Ciênc. Saúde Coletiva. 2007;12(5):1237-46.

31. Prado JS, Aquino DR, Cortelli JR, Cortelli SC. Condição dentária e hábitos de higiene bucal em crianças com idade escolar. Rev. biociênc. 2001;7(1):63-9.

32. Soares JMP, Aragão AKR, Jardim MCAM, et al. Gengivite e índice de higiene oral em crianças de Cabedelo-Paraíba - Brasil. Rev. bras. Cienc. Saúde. 2003;7(2):177-86.

Recebido em: 26/08/2015 / Aprovado em: 30/09/2015

Marcia Cristina Pereira de Souza Lima

Rua João Lisboa, 1091 - Centro

Imperatriz-MA, Brasil - CEP: 65.900-630

E-mail: marcia4000@globomail.com 